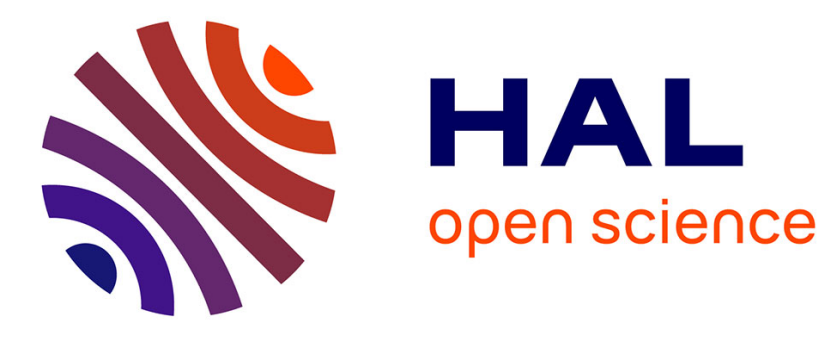

\title{
Predictive models for diffuse low-grade glioma patients under chemotherapy
}

\author{
Meriem Ben Abdallah, Marie Blonski, Sophie Wantz-Mézières, Yann \\ Gaudeau, Luc Taillandier, Jean-Marie Moureaux
}

\section{To cite this version:}

Meriem Ben Abdallah, Marie Blonski, Sophie Wantz-Mézières, Yann Gaudeau, Luc Taillandier, et al.. Predictive models for diffuse low-grade glioma patients under chemotherapy. 38th Annual International Conference of the IEEE Engineering in Medicine and Biology Society, EMBC'16, Aug 2016, Orlando, Florida, United States. hal-01316865

\section{HAL Id: hal-01316865 \\ https://hal.science/hal-01316865}

Submitted on 17 Nov 2016

HAL is a multi-disciplinary open access archive for the deposit and dissemination of scientific research documents, whether they are published or not. The documents may come from teaching and research institutions in France or abroad, or from public or private research centers.
L'archive ouverte pluridisciplinaire HAL, est destinée au dépôt et à la diffusion de documents scientifiques de niveau recherche, publiés ou non, émanant des établissements d'enseignement et de recherche français ou étrangers, des laboratoires publics ou privés. 


\title{
Predictive models for diffuse low-grade glioma patients under chemotherapy
}

\author{
Mériem Ben Abdallah*, Marie Blonski*†, Sophie Mézières ${ }^{\ddagger}$, Yann Gaudeau*§, \\ Luc Taillandier*广 and Jean-Marie Moureaux* \\ * Université de Lorraine, Centre de Recherche en Automatique de Nancy (CRAN), CNRS UMR 7039, \\ Faculté de Médecine - Bât D - BP 184, Vandoeuvre-lès-Nancy, 54505, France \\ $\dagger$ Neuro-Oncology Unit, Nancy University Hospital, Avenue du Maréchal Lattre de Tassigny, \\ 54035 Nancy, France \\ * Université de Lorraine, Institut de Mathématiques Elie Cartan, INRIA BIGS \\ CNRS UMR 7502, BP 239, F-54506 Vandoeuvre-lès-Nancy Cedex, France \\ $\S$ Université de Strasbourg, 30 Rue du Maire André Traband, Haguenau, 67500, France
}

\begin{abstract}
Diffuse low-grade gliomas are rare primitive cerebral tumours of adults. These tumors progress continuously over time and then turn to a higher grade of malignancy associated with neurological disability, leading ultimately to death. Tumour size is one of the most important prognostic factors. Thus, it is of great importance to be able to assess the volume of the tumor during the patients' monitoring. MRI is nowadays the recommended modality to achieve this. Furthermore, if surgery remains the first option for diffuse low-grade gliomas, chemotherapy is increasingly used (before or after a possible surgery). However, crucial and difficult questions remain to be answered: identifying subgroups of patients who could benefit from chemotherapy, determining the best time to initiate chemotherapy, defining the duration of chemotherapy and evaluating the optimal time to perform surgery, or otherwise radiotherapy. In this study, we propose to help clinicians in decision-making, by designing new predictive models dedicated to the evolution of the diameter of the tumor. Two proposed statistical models (linear and exponential) have been tested on a database of 16 patients whose temozolomidebased chemotherapy lasted between 14 and 32 months, with an average duration of $\mathbf{2 2 . 8 1 2 5}$ months. The selection of the most appropriate model has been achieved with the corrected Akaike's Information Criterion. The results are very promising, with coefficients of determination varying from 0.79 to 0.97 with an average value of 0.90 for the linear model. This shows it is possible to alert the clinician to a change in the tumor diameter's dynamics.
\end{abstract}

\section{INTRODUCTION}

Diffuse low-grade gliomas (DLGG) are rare and infiltrative brain tumors. Three phases characterize their evolution:

- The first phase is asymptomatic with a linear and constant tumor growth rate (about $3.5 \mathrm{~mm}$ per year) [1].

- The second phase is associated with few neurological symptoms (seizure most frequently) allied with a linear and constant tumor growth rate of about $4 \mathrm{~mm}$ per year [2].

- The third phase corresponds to the anaplastic transformation (high-grade glioma) with an increased tumor growth rate.

The aim of treatments is to delay as much as possible the anaplastic transformation while preserving the quality of life [3]. Functional surgery is the first therapeutic option because of its clear impact on survival while maintaining or improving the quality of life [4]. Radiotherapy, which was the first complementary treatment proposed, has the same impact, regardless of its realization's moment (earlier at diagnosis or later at progression) [5]. Furthermore, in an increasing manner, chemotherapy takes place earlier in the management of these patients, either in a neoadjuvant position (as a possible first treatment before surgery) or in case of progression after surgery for non re-operable patients [6][7]. It is now admitted that MRI is recommended in the monitoring of the DLGG patients [6] and allows the assessment of the tumor volume. Indeed, volume monitoring is essential in therapeutic decision. In this context, two techniques have been proposed in the literature to estimate the tumor volume : three diameters technique [2] and segmentation [1] which is considered a gold standard. Regardless of the adopted technique, one crucial issue is that the treatment strategy should be strictly tailored to each patient [8]. In the framework of chemotherapy, it is of high importance to be able to assess the impact and the expected benefit of the treatment in order to control it. Thus, modelling the response to chemotherapy would help clinicians in decision-making by identifying subgroups of patients who could benefit from chemotherapy, determining the best time to initiate chemotherapy, fixing the duration of chemotherapy and evaluating the optimal time to perform surgery. In other words, clinicians could more easily propose a tailored solution to each patient. To our knowledge, two recent studies have been conducted to address this issue and have offered interesting results [9][10]. The proposed solutions in these studies are based on a microscopic approach, at the cellular scale. Furthermore, the volume measurement is obtained by the three diameters method. Here, we propose a different approach at a macroscopic scale which is based on the design of two predictive models for DLGG patients under Temolozomide chemotherapy. Another key point lies in the tumor volume assessment which is based here on MRI scans' segmentation. Our models are data-driven and adapt 
in real-time to each new MRI. This makes our approach a practical tool in a clinical routine to assist, simply and quickly, doctors in the establishment of their treatment strategy. The two proposed models allow us to predict the tumor diameter as a variable evolving according to time and are based on a training set of 5 MRI acquisitions from the begining of chemotherapy. We show that if a new observed diameter (corresponding to the current MRI examination) is inside the prediction interval given by the model, a normal evolution can be considered. On the contrary, if the new observation leaves the prediction interval, a change in the tumor diameter's dynamics of evolution is foreseen and an alert can be given to the clinician to help him in decisionmaking.

The rest of the paper is organized as follows. In section 2, the material and method are presented. Models are detailed in section 3. Results are given in section 4. Finally, section 5 provides a discussion and a conclusion.

\section{MATERIALS AND METHODS}

Some DLGG patients have to undergo one or more chemotherapy sessions. After being confronted with the question of optimal timing of treatment initiation, physicians are then faced with the crucial choice of when to stop the treatment (if, of course, there is no tolerance problem). There is currently no consensus among the neuro-oncology community on how to determine that moment. In order to address this problem, we propose statistical predictive models which are based on the tumor diameter's evolution over time. This diameter is obtained from the tumor volume, that is numerically reconstructed from manual segmentations using the Delaunay-triangulation-based reconstruction algorithm of the OsiriX software. In this study, we will only consider patients in first-line chemotherapy, who didn't undergo any prior treatment, except surgery. We will also only investigate one type of chemotherapy, the temozolomide (TMZ), which is the most widely used molecule for DLGG due to its good tolerance by most patients and its practical use (oral treatment).

For this work, we tested multiple regression models to predict the moment to end chemotherapy. Spontaneously, without any treatment, DLGG tumor diameter evolves linearly over time [2]. We noticed that under chemotherapy, for some patients, the diameter evolves linearly, whereas, for other patients, the evolution is exponential. But for a small minority of cases, the evolution is neither monotonously decreasing nor even increasing and the curve does not fit any known or specific function. Our initial database included 21 patients who underwent TMZ chemotherapy. Five patients were excluded because they fitted neither the exponential nor the linear model. For these patients, and for other similar cases, we will adopt the classical approach of following up the dynamics of tumor growth. In the present article, we will only discuss the cases of patients following a linear or an exponential model, which represent the majority of our database (16/21 patients), and we will leave the discussion of other cases for later work. Our predictive models were thus applied on 16 patients whose duration of treatment lasted between 14 and 32 months, with an average duration of 22.8125 months. In this first study, we have included tumors regardless of their pathological (astrocytomas versus oligodendrogliomas versus mixed tumors) or molecular (including IDH $1 / 2$ or $1 \mathrm{p} 19 \mathrm{q})$ status.

\section{STATISTICAL ANALYSIS}

Let $D$ be the random variable representing the tumor diameter, following a simple linear regression model:

$$
D=b_{0}+b_{1} T+\varepsilon \quad \text { where }
$$

$T:$ the random variable representing the time of observation.

$\varepsilon:$ the unobserved error term.

$b_{0}$ : the initial value of the tumor diameter.

$b_{1}$ : the growth rate of the tumor diameter.

Let $D^{\prime}$ be the random variable representing the tumor diameter, following an asymptotic exponential model:

$$
D^{\prime}=a_{0}-a_{1} e^{-a_{2} T}+\varepsilon \quad \text { where }
$$

$a_{0}:$ the asymptotic term which ensures the data's adequacy with reality where the tumor diameter never equals zero, regardless of the chemotherapy's efficiency.

$a_{1}$ : the difference between the asymptotic term and the initial value of the tumor diameter.

$a_{2}$ : the decay rate constant.

Our prediction models resort to a training dataset of an iid sample $\left(D_{i}, T_{i}, \varepsilon_{i}\right)$ for the linear model and $\left(D_{i}^{\prime}, T_{i}, \varepsilon_{i}\right)$ for the exponential model, where $i$ is the observation number. Tumor diameters in the chemotherapy period, $\left(D_{i}\right)_{i=0 \ldots n}$ and $\left(D_{i}^{\prime}\right)_{i=0 \ldots n}$, are obtained from MRI scans' manual segmentation by a clinician expert using the OsiriX software. The number of available training dataset $n$ is low, often around 6 . In any case, to comply with our statistical analysis, $n$ must be at least equal to 5 . The first step is to estimate the parameters, $b_{0}$ and $b_{1}$ for the linear model, $a_{0}$, $a_{1}$ and $a_{2}$ for the exponential model, from the training data set.This estimation is based on the least squares method for the linear model and on the Gauss-Newton algorithm for the exponential model.

In both models, the statistical error terms $\varepsilon_{i}$ are assumed to be independent, normally distributed $\mathscr{N}(0, \sigma)$ and to fulfill the homoscedasticity condition. After estimating the parameters, we verify that these hypotheses are fullfilled. The statistical error terms $\varepsilon_{i}$ 's normality is verified using a Shapiro-Wilk test. The homoscedasticity condition is checked with a White test for the linear model and with the plot of the residuals vs. the fitted values for the exponential model. As for the independence hypothesis, a Durbin Watson test is used for the linear model and the plot of each residual vs. the previous residual is employed for the exponential model. Once these conditions are established, a Student test is applied on $b_{1}$ for the linear model, on $a_{1}$ and $a_{2}$ for the exponential model, in order to evaluate the significance of the regression. Moreover, the linear 
model's prediction quality is analysed with the coefficient of determination $R^{2}$.

If, for a given patient, all previous conditions are met for both models, we resort to the corrected Akaike's Information Criterion $\left(A I C_{c}\right)$ [11] to choose the model that best describes the dataset. $A I C_{c}$ is defined as follows:

$$
A I C_{c}=n \ln \frac{S S}{n}+2 K+\frac{2 K(K+1)}{n-K-1} \quad \text { where }
$$

$S S:$ the sum of the square of the vertical distances of the points from the curve .

$K:$ the number of parameters fit by the regression plus one. It equals 3 for the linear model and 4 for the exponential model.

The best model is the one with the minimum value of $A I C_{c}$. Once the model is validated, we agree the evolution of the tumor is either linear with estimated parameters $\hat{b}_{0}$, $\hat{b}_{1}$ or exponential with estimated parameters $\hat{a}_{0}, \hat{a}_{1}, \hat{a}_{2}$. Then a new observation is predicted at time $T_{n+1}$ as follows:

$$
\begin{aligned}
& \hat{D}_{n+1}=\hat{b}_{0}+\hat{b}_{1} T_{n+1} \quad \text { Linear model } \\
& \hat{D}_{n+1}^{\prime}=\hat{a}_{0}-\hat{a}_{1} e^{-\hat{a}_{2} T_{n+1}} \quad \text { Exponential model }
\end{aligned}
$$

A prediction interval is also defined for a certain type I error $\alpha$, providing the range of values in which $\hat{D}_{n+1}$ or $\hat{D}_{n+1}^{\prime}$ is likely to fall given $T_{n+1}$. If the new observation is inside the prediction interval, a normal evolution of the tumor diameter is announced. If it leaves the prediction interval, a change in the tumor diameter's dynamics of evolution is observed. This can be medically interpreted either positively (in case the diameter decreases below the lower endpoint of the prediction interval, heralding a likely good response to the treatment) or negatively (tumor growth under chemotherapy above the higher endpoint of prediction interval, patient not responding positively to the treatment).

\section{RESULTS}

The results of this study have been implemented with the $\mathrm{R}$ software. Among the 16 patients of our dataset, 13 are classified as linear and 3 are classified as exponential. We selected a type I error $\alpha$ equal to 0.1 . The coefficients of determination $R^{2}$ for the 13 linear patients vary from 0.77 to 0.97 with an average value of 0.90 . In this paper, we will present one example of our linear predictive model and another one for the exponential predictive model for a representative purpose.

\section{A. Example for the linear model}

Patient 1 underwent chemotherapy for 27 months. For the learning phase, we had 6 points and we predicted one point preceding the end of the chemotherapy. The estimation of the linear parameters $b_{0}$ and $b_{1}$ gave the values 5.268757 and -0.025601 respectively. We then tested the normality hypothesis with the Shapiro-Willk test: $W=$ $0.9888>$ W crit $=0.713$ for type I error equal to 0.01 . We validated the homoscedasticity condition with the White test: $f(2,3)=30.82>$ White $=3.7468$ for type I error equal to 0.01 . We also confirmed the non correlation hypothesis with the Durbin Watson test: $D W=1.9082$ which is between 1.5 and 2.5 as it should be to pass the test. We also verified the significance of the regression with a Student test on $b_{1}$. We then computed the coefficient of variation $R^{2}$ which is equal to 0.87 .

We also tested the exponential model. The exponential model coefficients $a_{0}, a_{1}$ and $a_{2}$ were estimated at $4.71256,0.61778$ and 0.08615304 respectively. We checked the normality hypothesis with the Shapiro-Wilk test: $W=$ $0.9021>$ W crit $=0.713$ for type I error equal to 0.01 . The homoscedasticity and the correlation conditions were verified with the corresponding plots. As for the significance of the regression, we confirmed it with a Student test on $a_{1}$ and $a_{2}$.

To choose the best model, we computed $A I C_{c}$ for both models. $A I C_{c_{\text {Linear }}}=-6.495425<A I C_{c_{\text {Exponential }}}=$ -1.268149 . We thus chose the linear model to predict the last value of the diameter before the completion of chemotherapy as well as the prediction interval for type I error $\alpha$ equal to 0.1 . The visualization of the curve for the training dataset, the real diameter value at next time and the prediction interval in fig. 1 show clearly that the data follows the regression line and that the real point for the predicted one is above the higher endpoint of the prediction interval. The displayed alert message is the following: "Significant increase in the diameter".

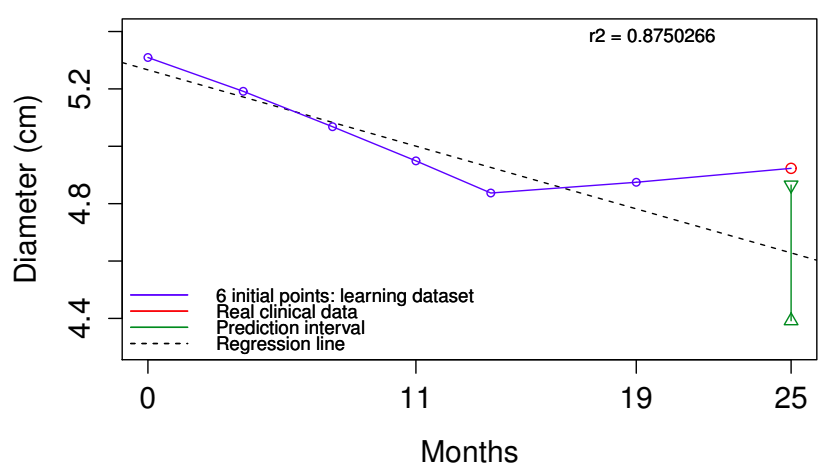

Fig. 1. The training dataset, the real value and the prediction interval with the linear model for patient 1 .

\section{B. Example for the exponential model}

Patient 2 underwent chemotherapy for 26 months. For the training phase, we had 6 points and we predicted one point preceding the end of the chemotherapy. Starting with the exponential model, the learning step returned the values 4.93097, 2.47087 and 0.1252605 for the estimation of $a_{0}$, $a_{1}$ and $a_{2}$ respectively. The normality hypothesis was tested with the Shapiro-Willk test: $W=0.8965>W$ crit $=0.713$ for type I error equal to 0.01 . The homoscedasticity and the correlation conditions were verified with the corresponding 
plots. As for the significance of the regression, we confirmed it with a Student test on $a_{1}$ and $a_{2}$. We also tested the linear model, but as the non correlation hypothesis was not confirmed $(D W=1.0995<1.5)$, this model was rejected.

With the selected exponential model, we finally estimated the last point preceding the end of the treatment as well as the prediction interval for type I error $\alpha$ equal to 0.1 . Fig.2 shows the curve for the training dataset, the real diameter value, the predicted diameter and the prediction interval. The real data seems to perfectly fit the exponential model and the real point is inside the prediction interval. The displayed alert message is the following: "Regular diameter evolution".

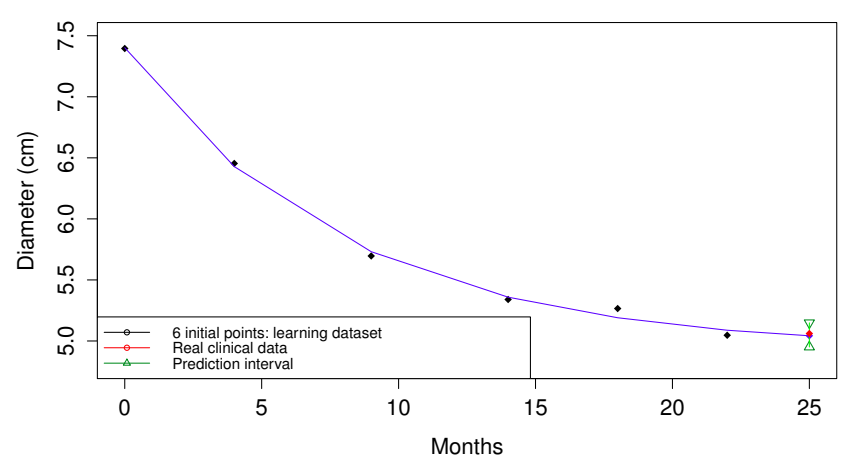

Fig. 2. The training dataset, the real value and the prediction interval with the exponential model for patient 2 .

\section{DISCUSSION AND CONCLUSION}

In this paper, we presented a new approach to help clinicians in decision-making for DLGG patients under chemotherapy. This approach is based on two statistical predictive models (linear and exponential) dedicated to the measure of tumor's diameter, as tumor size is one of the most important prognostic factors. Our study, conducted in Nancy University Hospital, France, relied on 16 patients whose temozolomide-based chemotherapy lasted between 14 and 32 months, with an average duration of 22.8125 . Within this database, 13 patients followed the linear model while the 3 others followed the exponential model. Based on a training set including at least 5 MRI scans, the model is able to predict the next diameter from the current one with significant accuracy. Thus, thanks to the corresponding computed prediction interval, it is possible to check if the actual measured diameter matches the predicted one. Then, if the actual diameter lies within the prediction interval, a "normal evolution" message is given. In the opposite case, the clinician is alerted by a "Significant increase in the diameter" message. We are aware that the models' validation should rely on a different dataset than the learning dataset. Nevertheless, the small number of cases has not allowed us to carry out this procedure. That is why, in order to increase the training set size, but also to allow our models to predict diameters sooner within the chemotherapy period, a new protocol has been established in Nancy University
Hospital. New patients who agree to this protocol benefit from additional non injected MRI scans at the begining of the treatment. Furthermore, in the near future, we intend to enlarge our DLGG database thanks to a collaboration with Montpellier University Hospital, France. Finally, as other molecular (IDH 1 or 2 mutations, 1p19q codeletion, MGMT promoter methylation, TERT promoter or ATRX mutations, etc.) or radiological (perfusion MRI, MR spectroscopy, amino acid PET imaging, etc.) factors could significantly influence tumor growth, we plan to include some of them in the design of our proposed models. Our long-term goal is to design a decision support tool which, based on different factors (tumor diameter, molecular and radiological parameters, etc.), would deliver a message to the clinician on the evolution of the DLGG in order to enable a personalized therapeutic management of patients.

\section{REFERENCES}

[1] J. Pallud, D. Fontaine, H. Duffau, E. Mandonnet, N. Sanai, et al., "Natural history of incidental world health organization grade II gliomas," Annals of Neurology, vol. 68 (5), pp. 727-733, Nov. 2010.

[2] E. Mandonnet, J.-Y. Delattre, M. L. Tanguy, K. R. Swanson, A. F. Carpentier, et al., "Continuous growth of mean tumor diameter in a subset of grade II gliomas," Annals of Neurology, vol. 53 (4), pp. 524-528, Apr. 2003.

[3] H. Duffau, "Preserving quality of life is not incompatible with increasing overall survival in diffuse low-grade glioma patients," Acta Neurochirurgica, vol. 157 (2), pp. 165-167, Feb. 2015.

[4] A. S. Jakola, K. S. Myrmel, R. Kloster, S. H. Torp, S. Lindal, et al., "Comparison of a strategy favoring early surgical resection vs a strategy favoring watchful waiting in low-grade gliomas," JAMA, vol. 308 (18), pp. 1881-1888, Nov. 2012.

[5] M. J. van den Bent, D. Afra, O. de Witte, M. ben Hassel, S. Schraub, et al., "Long-term efficacy of early versus delayed radiotherapy for low-grade astrocytoma and oligodendroglioma in adults: the eortc 22845 randomised trial," The Lancet, vol. 366 (9490), pp. 985-990, Sept. 2005 .

[6] R. Soffietti, B. G. Baumert, A. von Deimling, H. Duffau, M. Frénay, et al., "Guidelines on management of low-grade gliomas: report of an efns-eano task force," European Journal of Neurology, vol. 17 (9), pp. 1124-1133, Sept. 2010.

[7] M. Blonski, J. Pallud, C. Gozé, E. Mandonnet, V. Rigau, et al., "Neoadjuvant chemotherapy may optimize the extent of resection of world health organization grade II gliomas: a case series of 17 patients," Journal of Neuro-oncology, vol. 113 (2), pp. 267-275, June 2013.

[8] H. Duffau and L. Taillandier, "New concepts in the management of diffuse low-grade glioma: Proposal of a multistage and individualized therapeutic approach," Neuro-Oncology, vol. 17 (3), pp. 332-342, March 2015.

[9] B. Ribba, G. Kaloshi, M. Peyre, D. Ricard, V. Calvez, et al., "A tumor growth inhibition model for low-grade glioma treated with chemotherapy or radiotherapy," Clinical Cancer Research, vol. 18 (18), pp. 5071-5080, Sept. 2012.

[10] P. Mazzocco, C. Barthélémy, G. Kaloshi, M. Lavielle, D. Ricard, et al., "Prediction of response to temozolomide in low-grade glioma patients based on tumor size dynamics and genetic characteristics," CPT: Pharmacometrics \& Systems Pharmacology, vol. 4 (12), pp. 728-737, Dec. 2015.

[11] H. Motulsky and A. Christopoulos, Fitting Models to Biological Data using Linear and Nonlinear Regression : A practical guide to curve fitting. Oxford University Press, 2004. 